\title{
Suplementação de sulfato ferroso: prevenção da anemia ferropriva na faixa etária pediátrica
}

Ferrous sulfate supplementation: prevention of iron deficiency anemia in the pediatric age group

Suplementos de sulfato ferroso: prevención de la anemia por deficiencia de hierro en el grupo de edad pediátrica

Laís Assunção Vilefort ${ }^{1 *}$, Patrícia Pimenta Nunes ${ }^{1}$, Camila Campos Avelar Guimarães ${ }^{1}$, Rachel Pimentel Romano Silveira², Rayssa Mara Ferreira Costa ${ }^{3}$, Letícia Resende Sousa ${ }^{3}$, Sofia Lúcia El Hauche Pereira ${ }^{4}$, Karolyne Mariano Abrantes ${ }^{5}$, Maria Eduarda Galhardo Cabral ${ }^{1}$, Noelly Silva Borburema ${ }^{1}$.

\section{RESUMO}

Objetivo: Identificar os prejuízos causados pela anemia por deficiência de ferro na infância, chamando atenção para a importância e os benefícios da suplementação de sulfato ferroso nessa faixa etária como forma de prevenção da anemia ferropriva em crianças. Revisão bibliográfica: A anemia ferropriva é considerada um problema de saúde pública, sobretudo nos países em desenvolvimento. É consequência de uma das carências nutricionais mais comuns e afeta, principalmente, crianças com idade entre 6 e 24 meses, as quais apresentam risco duas vezes maior de desenvolverem a doença. Com o objetivo de reduzir a incidência da deficiência de ferro nessa população específica, os principais consensos de pediatria indicam a suplementação profilática de sulfato ferroso em crianças nessa faixa etária. Considerações finais: Um grande problema de saúde pública é a anemia causada por deficiência de ferro. Quando presente na infância é capaz de gerar inúmeros efeitos negativos no crescimento e desenvolvimento da criança. O diagnóstico e tratamento precoces desse distúrbio, assim como a suplementação profilática com sulfato ferroso a partir do $3^{\circ}$ mês de vida são medidas de extrema importância ca,,,pazes de melhorar de forma significativa os desfechos em saúde nessa área.

Palavras-chave: Sulfato ferroso, Suplementação, Pediatria, Anemia ferropriva.

\begin{abstract}
Objective: To identify the damage caused by iron deficiency anemia in childhood, drawing attention to the importance and benefits of ferrous sulfate supplementation in this age group as a way to prevent iron deficiency anemia in children. Bibligraphic review: Iron deficiency anemia is considered a public health problem, especially in developing countries. It is a consequence of one of the most common nutritional deficiencies and mainly affects children aged between 6 and 24 months, who are at twice the risk of developing the disease. In order to reduce the incidence of iron deficiency in this specific population, the main pediatric consensuses indicate the prophylactic supplementation of ferrous sulfate in children in this age group. Final considerations: Iron deficiency anemia is a serious public health problem. When present in childhood, it is capable of generating numerous negative effects on the child's growth and development. Early diagnosis and treatment of this disorder, as well as prophylactic supplementation with ferrous sulfate from the 3rd month of life onwards, are extremely important measures capable of significantly improving health outcomes in this area.
\end{abstract}

Key words: Ferrous sulfate, Supplementation, Pediatrics, Iron deficiency anemia.

\footnotetext{
${ }^{1}$ Faculdade de Minas de Belo Horizonte (FAMINAS-BH), Belo Horizonte - MG.

*E-mail: lavilefort@gmail.com

2 Universidade de Itaúna (UIT), Itaúna - MG.

${ }^{3}$ Centro Universitário Presidente Tancredo de Almeida Neves (UNIPTAN), São João Del Rei - MG.

${ }^{4}$ Faculdade de Medicina de Barbacena (FAME/FUNJOB), Barbacena - MG.

${ }^{5}$ Centro Universitário Facisa (Unifacisa), Campina Grande - PB.
} 


\section{RESUMEN}

Objetivo: Identificar el daño causado por la anemia ferropénica en la infancia, llamando la atención sobre la importancia y los beneficios de la suplementación con sulfato ferroso en este grupo de edad como una forma de prevenir la anemia ferropénica en los niños. Revisión bibliográfica: La anemia por deficiencia de hierro se considera un problema de salud pública, especialmente en los países en desarrollo. Es consecuencia de una de las deficiencias nutricionales más comunes y afecta principalmente a niños de entre 6 y 24 meses, que tienen el doble de riesgo de desarrollar la enfermedad. Para reducir la incidencia de deficiencia de hierro en esta población específica, los principales consensos pediátricos indican la suplementación profiláctica de sulfato ferroso en niños de este grupo de edad. Consideraciones finales: La anemia por deficiencia de hierro es un grave problema de salud pública. Cuando está presente en la infancia, es capaz de generar numerosos efectos negativos sobre el crecimiento y desarrollo del niño. El diagnóstico y tratamiento precoces de este trastorno, así como la suplementación profiláctica con sulfato ferroso a partir del tercer mes de vida, son medidas extremadamente importantes capaces de mejorar significativamente los resultados de salud en esta área.

Palabras clave: Sulfato ferroso, Suplementación, Pediatría, Anemia ferropénica.

\section{INTRODUÇÃO}

A anemia é definida, de acordo com a Organização Mundial da Saúde (OMS), como uma condição clínica em que a concentração de hemoglobina presente na corrente sanguínea se encontra em níveis inferiores aos valores de referência para idade e sexo, sendo insuficiente para suprir as necessidades básicas e fisiológicas do organismo (SOCIEDADE BRASILEIRA DE PEDIATRIA, 2018). Possui origem multifatorial, incluindo deficiência nutricional, infecções, perdas sanguíneas agudas ou crônicas e distúrbios genéticos, sendo a deficiência de ferro a causa mais comum (WANG M, 2016).

O ferro é o metal mais presente no organismo, fazendo parte da síntese proteica e dos sistemas respiratórios, oxidativos e anti-infecciosos. A hemoglobina contém aproximadamente $65-75 \%$ do ferro corporal total na forma de heme, outros $10-20 \%$ são armazenados na forma de ferritina e hemossiderina, cerca de $4 \%$ está contido em mioglobina, $3-4 \%$ em diversos sistemas enzimáticos e cerca de $2 \%$ em espécies reativas de oxigênio (MILLER JL, 2013).

Sua maior parcela provém do sistema de reciclagem de hemácias e apenas uma pequena quantidade é adquirida através da dieta por meio de fontes vegetais ou inorgânicas (ferro não hemínico) ou por meio de carnes e ovos (ferro hemínico ou orgânico) (SOCIEDADE BRASILEIRA DE PEDIATRIA, 2018). Diariamente uma pequena quantidade (1-2 mg/dia) é absorvida através da dieta, com objetivo de compensar perdas gastrointestinais e outras perdas de ferro, como suor e descamação da pele (MANTADAKIS E, et al., 2020).

Atualmentecalcula-se que cerca de $27 \%$ a $50 \%$ da população global seja acometida por deficiência de ferro e aproximadamente dois bilhões de pessoas no mundo apresentam anemia (SOCIEDADE BRASILEIRA DE PEDIATRIA, 2018). Dentre as pessoas acometidas, a grande maioria é de baixa renda e moram em locais de poucos recursos. Deficiências nutricionais de ferro, ácido fólico ou vitaminas, doenças crônicas, infecções parasitárias e envenenamento por chumbo são exemplos de fatores de riscos para quadros de anemia em países de baixa renda (MANTADAKIS E, et al., 2020).

Nos países desenvolvidos existem alguns grupos considerados de alto risco para o surgimento de anemia por deficiência de ferro, sendo eles bebês, crianças, adolescentes do sexo feminino, mulheres em idade reprodutiva e idosos. Já nos países chamados de industrializados, devido à alimentação precária, baseada em fast-foods, a única deficiência de micronutrientecomum de acontecer é a deficiência de ferro (MANTADAKIS E, et al., 2020).

A anemia ferroprivaocorre por uma produção insuficiente de hemoglobina devido a falhas no estoque de ferro e está diretamente relacionadaa deficiência de ferro não tratada. Quando essadeficiência édiagnosticada em um paciente, deve ser iniciado o tratamento o mais precoce possível para que sejam reduzidos os riscos de isquemia de órgãos ou piora do quadro de anemia. Caso o tratamento não seja iniciado, deve-se ao 
menosidentificar a causa e reduzi-la ou realizar o reabastecimento nos estoques de ferro da medula óssea. É importante ressaltar que essa conduta independe da presença de sintomas (KHAN L, 2018).

A deficiência de ferro em crianças também deve ser precocemente tratada, uma vez que está associada a deficiências neurológicas e cognitivas, à capacidade de aprendizagem reduzida e função motora prejudicada. Algumas manifestações clínicas muito comuns em pacientes com essa deficiência são síndrome das pernas inquietas, convulsões febris e crises de apneia. Fadiga e intolerância ao frio podem aparecer em adolescentes e mulheres adultas jovens e pode ser aliviada com a reposição oral adequada de ferro (CAPPELLINI MD, et al., 2019).

Segundo a OMS, anemia é definida como um problema de saúde pública leve quando a sua prevalência é de cerca de 5 a $20 \%$, moderadaquando é de 20 a $40 \%$ e grave quando se estende a mais de $40 \%$ da população. A maior parte dos países possui histórico de anemia como um problema de saúde pública considerado moderado agrave, isto é, mais de $20 \%$ da sociedade acometida (SOLIMAN AT, et al., 2017). Na infância, a anemia possui alto potencial de prejudicar o desenvolvimento mental e psicomotor da criança, de aumentar a morbimortalidade infantil, de causar queda no desempenho escolar e redução da resistência às infecções (ROBERT T, 2020).

O objetivo deste estudo foi identificar os prejuízos causados pela anemia por deficiência de ferro na infância, chamando atenção para a importância e os benefícios da suplementação de sulfato ferroso nessa faixa etária como forma de prevenção da anemia ferropriva em crianças.

\section{REVISÃO BIBLIOGRÁFICA}

\section{Papel do ferro no organismo humano}

Existem duas formas principaisde como ocorre a deficiência de ferro no organismo: a deficiência absoluta e a deficiência funcional. A chamada de absoluta ocorre por diminuição nos estoques de ferro do corpo. Já a conhecida como funcional possui os estoques totais de ferro normais ou aumentados, poréma medula óssea possui suprimento inadequado dessa molécula deixando prejudicada a produção dos glóbulos vermelhos. Existem algumas condições inflamatórias crônicas que frequentemente podem estar associada a anemia por deficiência de ferro, o que inclui doença inflamatória intestinal, doença hepática crônica, obesidade, insuficiência cardíaca crônica, doença renal crônicas e insuficiência cardíaca crônica (CAPPELLINI MD, et al., 2019).

Existem as chamadas proteínas heme, que faze parte da composição da hemoglobina e da mioglobina, que são responsáveis pelo transporte e armazenamento de oxigênio, se tornando essenciais para o funcionamento de todos os órgãos do corpo. O ferro é derivado dessas proteínas e também está presente em enzimas heme, enzimas de ferro não heme e proteínas de ferro-enxofre. O excesso de ferro deixa uma fração do ferro livre, conhecido como ferro "lábil", o que acaba gerando espécies reativas de oxigênio, causando estresse oxidativo no organismo. No corpo humano não há um sistema de excreção de ferro, o quefaz com que sua homeostase seja rigidamente regulada por meio de uma rede de proteínas responsáveis por seu armazenamento, exportação e transporte no corpo (UEDA N e TAKASAWA K, 2018).

\section{Repercussões da anemia ferropriva no organismo humano}

A anemia ferropriva, principalmente quando presente em crianças, tem um grande potencial de afetar o crescimento e comprometer o desenvolvimento de habilidades cognitivas, comportamentais e motoras, causando grandes repercussões ao longo da vida mesmo após o tratamento adequado (SOCIEDADE BRASILEIRA DE PEDIATRIA, 2018).

Além do comprometimento do crescimento e desenvolvimento, a carência de ferro na infância também pode predispor ao surgimento de cáries dentárias, redução da capacidade de discriminar e identificar odores, alterações de paladar e apetite (associação a quadros de pica), queda da imunidade, alteração da resposta ao estresse metabólico e alterações do desenvolvimento audiovisual (MATTIELLO V, et al., 2020). 
Sabe-se também que a existência de anemia durante a gestação está diretamente associada à ocorrência de prematuridade, baixo peso ao nascimento e aumento da morbimortalidade perinatal e neonatal. Segundo pesquisas, a anemia materna é capaz de influenciar nos valores de hemoglobina da criança aos 6 meses de vida, mesmo em aleitamento materno exclusivo (SOCIEDADE BRASILEIRA DE PEDIATRIA, 2018).

\section{Fatores de risco e prevenção para anemia ferropriva}

A principal causa de anemia ferropriva em países em desenvolvimento é a alimentação baseada em dietas com baixa biodisponibilidade de ferro. As dietas são, predominantemente, baseadas em farinhas à base de cereais ou leguminosas na maior parte dos países de baixa renda, enquanto o consumo dealimentos ricos em ferro e zinco, como carnes, aves e peixes, é comumente reduzido por razões econômicas, culturais ou religiosas. Como os alimentos com alta biodisponibilidade de ferro são de origem animal eles possuem um custo mais elevado e uma disponibilidade mais escassa, o que torna mais difícil manter um equilíbrio adequado de ferro em ambientes com recursos limitados. Nesses países são encontrados fatores de risco para a deficiência de ferro devido ao consumo reduzido de carne vermelha, o consumo frequente de chá, a ingestão inadequada de vitamina $\mathrm{C}$ e o alto consumo dietético de fitatos e polifenóis (WARNER MJ e KAMRAN MT, 2020).

Perda de sangue gastrointestinal e genital, assim como erros alimentares são as etiologias mais comuns da anemia ferropriva em países desenvolvidos. Algumas práticas altamente associadas à deficiência de ferro em países industrializados incluem hábitos alimentares incorretos, redução do consumo de leite fortificado com ferro, amamentação prolongada sem suplementação de ferro após o quarto mês de vida, introdução de leite de vaca antes de um ano de idade ou com consumo superior a $500 \mathrm{~mL} / \mathrm{dia}$, consumo reduzido de carne vermelha e dietas veganas (CAMASCHELLA C, 2019).

Além disso, podem ser citadas como fatores de risco adicionais para a deficiência desse micronutriente a presença de doença celíaca, a giardíase sintomática, a gastrectomia, a diminuição da acidez gástrica, a ingestão oral inadequada de ferro por razões religiosas ou culturais, fluxo menstrual prolongado ou itenso, uso de dispositivos intrauterinos como anticoncepcionais, infecções de longa duração por Helicobacter pylori, ancilostomíase, esquistossomose, perda de sangue traumática ou operatória, doação de sangue, distúrbios hemorrágicos e sangramento gastrointestinal devido ao uso de antitrombótico, antiplaquetário ou antiinflamatório não esteroidal (CAMASCHELLA C, 2019).

\section{Diagnóstico clínico-laboratorial da anemia ferropriva}

As manifestações clínicas geradas por uma anemia decorrente da deficiência de ferro são determinadas pelo grau de depleção desse micronutriente no organismo e sua repercussão na produção de hemoglobina. Dentre as manifestações mais comuns estão fadiga, apatia, baixo ganho pôndero-estatural, unhas fracas e quebradiças, palidez, irritabilidade e taquicardia. Outros sinais são redução da acidez gástrica, gastrite atrófica, sangramento da mucosa intestinal e estomatite angular (SOLIMAN AT, et al., 2017).

Existem alguns critérios laboratoriais que podem ser considerados para se realizar o diagnóstico de uma anemia, mas, em geral, uma hemoglobina baixa é o principal indicador (SHORT MW e DOMAGALSKI JE, 2013). Para diagnóstico de anemia, os valores da concentração de hemoglobina na corrente sanguínea considerados pela OMS são: inferiores a 11 g/dl para crianças de 6 a 60 meses e inferiores a 11,5g/dl para crianças de 5 a 11 anos de idade (SOCIEDADE BRASILEIRA DE PEDIATRIA, 2018).

Existem estágios de depleção do ferro que podem ser identificados através de exames específicos, sendo a anemia ferropriva o estágio final da deficiência de ferro (BOURI S e MARTIN J, 2018).O primeiro estágio é caracterizado por uma redução dos estoques, sendo refletida pela redução dos níveis de ferritina sérica. $O$ segundo estágio é caracterizado pela redução do ferro sérico, aumento da capacidade total de ligação da transferrina e diminuição do índice de saturação da transferrina. O terceiro e último estágio é quando o ferro é insuficiente para eritropoese, gerando a anemia propriamente dita. Outros exames da cinética do ferro podem ser solicitados para análise completa do quadro comotransferrina, zincoprotoporfirina eritrocitária e capacidade total de ligação do ferro (SOCIEDADE BRASILEIRA DE PEDIATRIA, 2018). 


\section{Tratamento da anemia ferropriva em crianças}

Um ponto de extrema importância no tratamento da anemia é identificar a sua causa. Trata-se de uma doença predominante em países subdesenvolvidos onde dentre as principais causas estão deficiências nutricionais (ferro, vitamina B12, ácido fólico, riboflavina), doenças crônicas, infecções parasitárias como malária, hemoglobinopatias e envenenamento por chumbo (MANTADAKIS E, et al.,2020).

O tratamento da anemia ferropriva deve ser realizado através de uma adequação alimentar e reposição oral de ferro. Para crianças a recomendação é de $3-5 \mathrm{mg}$ de ferro elementar $/ \mathrm{kg} / \mathrm{dia}$, por pelo menos oito semanas, podendo se estender por até 3-6 meses a depender da resposta (SOCIEDADE DE PEDIATRIA DE SÃO PAULO, 2019).

Os sais ferrosos (sulfato ferroso, fumarato ferroso e gluconato ferroso) são eficazes para reposição de ferro, mas apresentam efeitos colaterais gastrointestinais como náuseas, vômitos, diarreia, obstipação, pirose, plenitude, dispepsia, gosto metálico e desconforto abdominal. Sendo assim a dose considerada ideal pode variar de acordo com a tolerância do paciente e a recomendação é que sejam ofertados longe das refeições e em jejum (SOCIEDADE BRASILEIRA DE PEDIATRIA, 2018).

Os sais férricos e amino-quelatos também podem ser utilizados para o tratamento e a vantagem é que possuem menos efeitos adversos, proporcionando uma melhor adesão ao tratamento. Outras opções são o ferro carbolina e ferro quelato, que assim como os sais férricos possuem maior tolerância e não sofrem influência da dieta na absorção (SOCIEDADE DE PEDIATRIA DE SÃO PAULO, 2019). Pra a correção da anemia ferropriva a eficácia dos sais férricos é semelhante a dos ferrosos (SOCIEDADE BRASILEIRA DE PEDIATRIA, 2018).

A administração de ferro parenteral tem indicações restritas a situações de anemia grave: falha terapêutica por via oral, refratariedade, intolerância que impede o uso oral, falha de absorção (distúrbios do trato gastrointestinal), grandes perdas sanguíneas, doenças inflamatórias crônicas, doença renal crônica, quimioterapia dentre outras situações (SOCIEDADE DE PEDIATRIA DE SÃO PAULO, 2019).

\section{Suplementação profilática de ferro em crianças}

As ações de educação alimentar voltadas para a prevenção da anemia ferropriva preconizam o aleitamento materno exclusivo e prolongado e introdução de alimentos ricos em ferro no período adequado. A contraindicação do leite de vaca in natura antes dos 12 meses de idade também atua como uma estratégia protetora contra a deficiência de ferro e consequente desenvolvimento de anemia ferropriva(MATTIELLO V, et al., 2020)

O Brasil apresenta uma política para a suplementação do ferro desde 2005 (Programa Nacional de Suplementação de Ferro (PNSF). A Sociedade Brasileira de Pediatria (SBP) orienta que a suplementação profilática de ferro é indicada para todos os recém-nascidos a termo, de peso adequado para a idade gestacional, com $1 \mathrm{mg}$ de ferro elementar $/ \mathrm{kg} / \mathrm{dia}$ a partir do $3^{\circ}$ mês (independentemente da forma de aleitamento) até $02^{\circ}$ ano de vida. Em todos os casos a suplementação é reduzida após o $1^{\circ}$ ano de vida, sendo passada, por mais 12 meses, para uma dose de $1 \mathrm{mg} / \mathrm{kg} / \mathrm{dia}$ (SOCIEDADE BRASILEIRA DE PEDIATRIA, 2018).

As doses variam de acordo com o peso e a idade gestacional do bebê. A suplementação segue as seguintes posologias: para o recém-nascido (RN) termo, com peso adequado em aleitamento exclusivo ou não ou recebendo menor a dose é de $1 \mathrm{mg}$ de ferro elementar/kg de peso/dia dos 3 aos 24 meses. Para o RN termo, porém com peso inferior a $2.500 \mathrm{~g}$ e para o RN pré-termo com peso entre $1.500 \mathrm{~g}$ e $2.500 \mathrm{~g} \mathrm{a}$ suplementação é feita com $2 \mathrm{mg}$ de ferro elementar/kg de peso/dia a partir de 30 dias de vida, pelo período de 1 ano e, posteriormente, com $1 \mathrm{mg}$ de ferro elementar/kg de peso/ dia por mais 1 ano (SOCIEDADE DE PEDIATRIA DE SÃO PAULO, 2019).

O RN pré-termo com peso entre $1.000 \mathrm{~g}$ e $1.500 \mathrm{~g}$ deverá receber $3 \mathrm{mg}$ de ferro elementar $/ \mathrm{kg}$ de peso/dia a partir de 30 dias de vida por 1 ano e RN pré-termo com peso menor que $1.000 \mathrm{~g}$, necessita de uma dose 
ainda maior, $4 \mathrm{mg}$ de ferro elementar $/ \mathrm{kg}$ de peso/dia a partir de 30 dias de vida durante 1 ano, posteriormente, ambos irão receber $1 \mathrm{mg}$ de ferro elementar $/ \mathrm{kg}$ de peso/dia por mais 1 ano. É importante ressaltar que a dose de ferro elementar necessita ser calculado de acordo com a apresentação do medicamento que está sendo usado (SOCIEDADE DE PEDIATRIA DE SÃO PAULO, 2019)

A suplementação medicamentosa deve ser sempre acompanhada de uma alimentação adequada na infância, portanto é indicado o aleitamento materno exclusivo até os seis meses de vida, mantendo-se até os dois anos de idade ou mais, além de uma adequada introdução alimentar, a partir do sexto mês de vida, por meio de alimentos que aumentem a biodisponibilidade e absorção do ferro (SOCIEDADE DE PEDIATRIA DE SÃO PAULO, 2019).

A anemia ferropriva é a forma mais comum de anemia no mundo, atingindo milhares de crianças e adultos. É mais comum em países subdesenvolvidos, onde as diversas condições econômico-sociais propiciam o desenvolvimento dessa doença(CAPPELLINI MD, et al., 2019).

O diagnóstico da anemia por deficiência de ferro é predominantemente laboratorial, guiada por alterações presentes no hemograma, sendo o principal indicador a queda da hemoglobina e por alterações dos exames da cinética do ferro. Manifestações clínicas podem ocorrer a depender do grau de deficiência e grau da anemia, no entanto são em sua maioria inespecíficos e insuficientes para fundamentar um diagnóstico preciso sem necessidade de complemento(ROBERT T, 2020).

O tratamento da deficiência de ferro pode ser realizado através da suplementação oral de ferro por meio de sais ferrosos, sais férricos, ferrocarbolina e ferro quelato. A suplementação por via venosa é reservada apenas para casos específicos. A suplementação é fortemente recomendada pelas Sociedades de Pediatria do $3^{\circ}$ ao $24^{\circ}$ de vida com ferro via oral (SOCIEDADE DE PEDIATRIA DE SÃO PAULO, 2019).

\section{CONSIDERAÇÕES FINAIS}

A anemia por deficiência de ferro é um grave problema de saúde pública predominante nos países em desenvolvimento, mas que ocorre também nos países desenvolvidos. Quando presente na infância é capaz de gerar inúmeros efeitos negativos no crescimento e desenvolvimento da criança, como predispor ao surgimento de doenças e prejudicar funções neurocognitivas. O diagnóstico desse tipo de anemia é realizado principalmente através dos valores reduzidos de hemoglobina para idade e sexo e a causa é determinada através da alteração dos exames que compõe a cinética do ferro como ferro sérico, ferritina, cinética do ferro e índice de saturação da transferrina. $O$ tratamento precoce desse distúrbio quando presente, assim como a suplementação profilática com sulfato ferroso a partir do $3^{\circ}$ mês de vida são medidas de extrema importância capazes de melhorar de forma significativa os desfechos em saúde nessa área.

\section{REFERÊNCIAS}

1. BOURI S, MARTIN J. Investigation of iron deficiency anaemia. Clinical Medicine, 2018; 18(3): 242-244.

2. CAMASCHELLA C. Iron deficiency. Blood, 2019; 133(1): 30-39.

1. 3.CAPPELLINI MD, et al. Iron deficiency anaemia revisited. Journal of Internal Medicine, 2019; 287(2): $153-170$.

3. KHAN L. Anemia in Childhood. PediatricAnnals, 2018; 47(2): e42-e47

4. MANTADAKIS E, et al. Iron Deficiency Anemia in Children Residing in High and Low-Income Countries: Risk Factors, Prevention, Diagnosis and Therapy. Mediterranean Journal of Hematology and Infectious Diseases, 2020; 12(1): e2020041.

5. MATTIELLO V, et al. Diagnosis and management of iron deficiency in children with or without anemia: consensus recommendations of the SPOG Pediatric Hematology Working Grouop. European Journal of Pediatrics, 2020; 179(4): 527-545.

6. MEANS RT. Iron Deficiency and Iron Deficiency Anemia: Implications and Impact in Pregnancy, Fetal Development, and Early Childhood Parameters. Nutrients, 2020; 12(2): 447.

7. MILLER JL. Iron deficiency anemia: a common and curable disease.Cold Spring Harbor Perspectives in Medicine, 2013; 3(7): a011866.

8. SHORT MW, DOMAGALSKI JE. Iron deficiency anemia: evaluation and management. American Academy of Family Physicians, 2013; 87(2): 98-104. 
9. SOCIEDADE BRASILEIRA DE PEDIATRIA. Consenso sobre anemia ferropriva: mais que uma doença, uma urgência médica!. Diretrizes Departamentos de Nutrologia e Hematologia-Hemoterapia, 2018; 1(2):1-13. Disponível em: https://www.sbp.com.br/fileadmin/user_upload/21019f-Diretrizes_Consenso_sobre_anemia_ferropriva-ok.pdf. Acesso em: 2 ago. 2021.

10. SOCIEDADE DE PEDIATRIA DE SÃO PAULO. Suplementação de nutrientes. Pediatria atualize-se, boletim da SBSP, 2019; 4(5):4-10. Disponível em: https://www.spsp.org.br/site/asp/boletins/AtualizeA4N5.pdf. Acesso em: 02 ago. 2021.

11. SOLIMAN AT, et al. Iron deficiency anemia and glucose metabolism. Acta Biomedica, 2017; 88(1): 112-118.

12. SUNDARARAJAN S, RABE H. Prevention of iron deficiency anemia in infants and toddlers. Pediatric Research, 2021; 89(1): 63-73.

13. UEDA N, TAKASAWA K. Impact of Inflammation on Ferritin, Hepcidin and the Management of Iron Deficiency Anemia in Chronic Kidney Disease. Nutrients, 2018; 10(9): 1173.

14. WANG M. Iron Deficiency and Other Types of Anemia in Infants and Children. American Academy of Family Physicians, 2016; 93(4): 270-8.

15. WARNER MJ, KAMRAN MT. Iron Deficiency Anemia. StatPearls Publishing LLC, 2020; 1(2): 1-4. 